\title{
Diagnóstico nutricional en pacientes con mielomeningocele por nivel de lesión
}

\author{
García-Guerrero PL*, Rodríguez-Hernández Al**, Galván-Guerra M ${ }^{* * *}$, Solís-Arias $R^{* * *}$
}

- Resumen
- grave de la columna vertebral. La mayoría de los pa-
- de Rehabilitación Infantil Teletón Aguascalientes (CRIT),
- de lesión y discapacidad, lo cual puede influir en el
- la prevalencia de obesidad y/o sobrepeso en el grupo
- de pacientes con mielomeningocele, se estudiaron 91
- del CRIT Aguascalieron a la Clínica de lesión medular
- de la población fue diagnostramos que el $38.5 \%$
- ton con algún grado

Palabras clave: sobrepeso, obesidad, mielomeningocele, tipo de lesión medular.

\section{Introducción}

El mielomeningocele $(M M)$ es la malformación congénita más grave de la columna vertebral, corresponde a un defecto en el desarrollo de las apófisis laminares, las cuales no se fusionan en la línea media para formar una apófisis espinosa dorsal única, lo cual produce distensión quística de las meninges y anormalidades estructurales o funcionales de la médula espinal o de la cauda equina de manera secundaria. ${ }^{1}$ El MM causa anormalidades neurológi-

* Pasante de Servicio Social en el Centro de Rehabilitación Infantil Teletón

** Maestro en Nutrición Clínica del CRIT Aguascalientes

*** Médicos especialistas en Rehabilitación CRIT Aguascalientes

Fecha de recibido 13 agosto 2010

- Fecha de aceptación 13 diciembre 2010

Correspondencia Adán Israel Rodríguez Hernández, Centro de Rehabilitación Infantil Teletón Ags., Heroico Colegio Militar y VázquezCol Santa Anita 2a Sección Ags., Ags. adan_israelll@yahoo.com.mx 


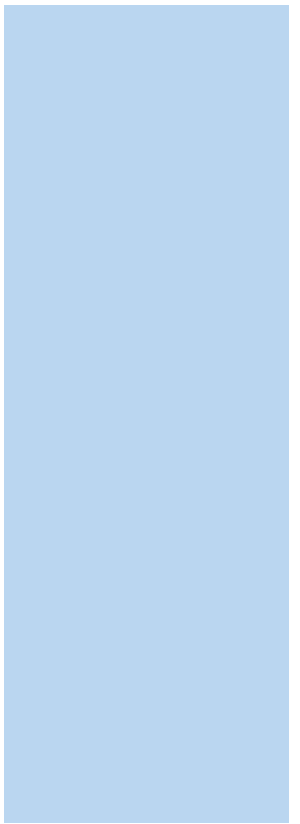

cas graves y permanentes; es responsable de aproximadamente el $75 \%$ de todos los casos de espina bífida descritos en la literatura. Los niños afectados con esta deformación necesitarán el cuidado de un equipo médico por el resto de su vida. ${ }^{2}$ En México existen diferentes datos acerca de la incidencia de $M M$ en la población infantil, de acuerdo a un estudio realizado en el Instituto Nacional de Pediatría en la ciudad de México, la frecuencia es de 1.2 de cada mil nacimientos, aunque otros autores consideran una prevalencia de 2 afectados por cada mil. En general se considera que la incidencia es de 1 afectado por cada 700/800 nacimientos. ${ }^{3}$ En diversos estudios $^{3,4}$ se expone el sobrepeso y la obesidad como el problema nutricional más frecuente en pacientes con mielomeningocele y se da énfasis a la importancia de la implementación de programas que aseguren la vigilancia a nivel nutricional de estos pacientes, de manera que se pueda mejorar su calidad de vida. El objetivo de este estudio fue conocer la prevalencia de sobrepeso y obesidad en el grupo de pacientes atendidos en la clínica de lesión medular del CRIT Aguascalientes.

\section{| | | | | | | | | | | | | | | | | | | | | | | | | | | | | | | | | | | | | | | | | | | | | | | | | | | | | | | | | | | | | | | | | | | | | | | | | | | | | | | | | | | | | | | | | | | | | | | | | | | ||}

\section{Material y métodos}

Se realizó un estudio retrospectivo de una serie de casos, donde se analizó la información correspondiente a un universo de 91 pacientes de la clínica de lesión medular del CRIT Aguascalientes, con diagnóstico de mielomeningocele, que acudieron a la consulta de Nutrición en el turno matutino, de enero a diciembre de 2008. Se clasificó a los pacientes con secuelas de mielomeningocele de acuerdo a la ubicación de la lesión; el nivel de lesión se definió como la zona de la columna vertebral donde iniciaba la malformación que podía ser a nivel dorsal, lumbar o sacro. También se clasifi- có a los pacientes por grupos de edad en: edad preescolar, escolar y adolescentes, así como por género.

Se evaluó el estado nutricional de noventa y un pacientes con mielomeningocele. Para establecer el estado nutricional de los pacientes, se utilizó como criterio diagnóstico el porcentaje estándar de peso, se compararon el peso y la talla en las tablas de la CDC, para poder obtener el peso ideal de acuerdo a su índice de masa corporal (IMC). La captura y análisis de los datos se realizó mediante los programas DBASE y SPSS, únicamente se realizó estadística descriptiva.

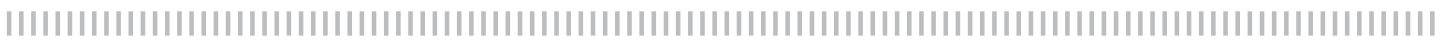

\section{Resultados}

Se estudiaron 91 pacientes con mielomeningocele, de los cuales 47 fueron hombres distribución según la edad fue: 17 (18.7\%) $(51.6 \%)$ y $44(48.4 \%)$ fueron mujeres. Su preescolares, $46(50.5 \%)$ escolares y 28 (30.8\%) adolescentes. Ver Tabla 1. 


\section{Tabla I}

\section{Características clínicas de 91 pacientes con mielomeningocele del CRIT Aguascalientes de enero a} diciembre de 2008.

\begin{tabular}{|c|c|c|}
\hline Característica & Número & Porcentaje \\
\hline \multicolumn{3}{|l|}{ Género } \\
\hline Masculino & 47 & 51.6 \\
\hline Femenino & 44 & 48.4 \\
\hline \multicolumn{3}{|l|}{ Grupo de edad } \\
\hline Preescolar & 17 & 18.7 \\
\hline Escolar & 46 & 50.5 \\
\hline Adolescente & 28 & 8.8 \\
\hline \multicolumn{3}{|l|}{ Nivel de lesión } \\
\hline Lumbar & 66 & 72.5 \\
\hline Dorsal & 16 & 17.6 \\
\hline Sacra & 9 & 9.9 \\
\hline
\end{tabular}

De los 91 pacientes, en $9(9.9 \%)$ se encontró desnutrición leve, 33 (36.3\%) presentaron peso y talla normal para la edad, en $14(15.2 \%)$ se observó sobrepeso, obesidad grado I en $15(16.5 \%)$, obesidad grado 2 en $8(8.8 \%)$, obesidad grado 3 en $4(4.5 \%)$ y en $8(8.8 \%)$ obesidad mórbida. Es decir el 53.8\% de la población estudiada presentó algún grado de sobrepeso u obesidad (Gráfica 1). En la mayoría de los pacientes la lesión se presentó a nivel lumbar (36.3\%) seguida de lesión dorsal (17.6\%) y por último la lesión sacra (9.9\%) (Gráfica 2)

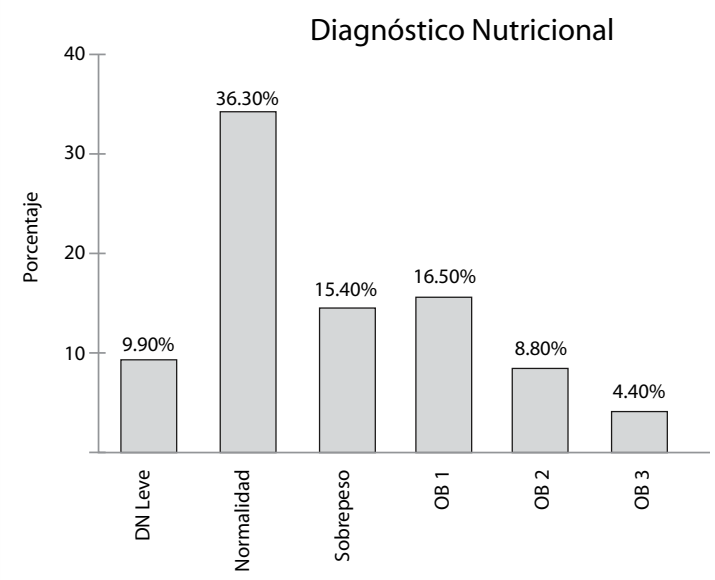

Gráfica 1. Diagnóstico nutricio en 91 pacientes con mielomeningocele del CRIT Aguascalientes de enero a diciembre de 2008. (DN=Desnutrición, OB=Obesidad)

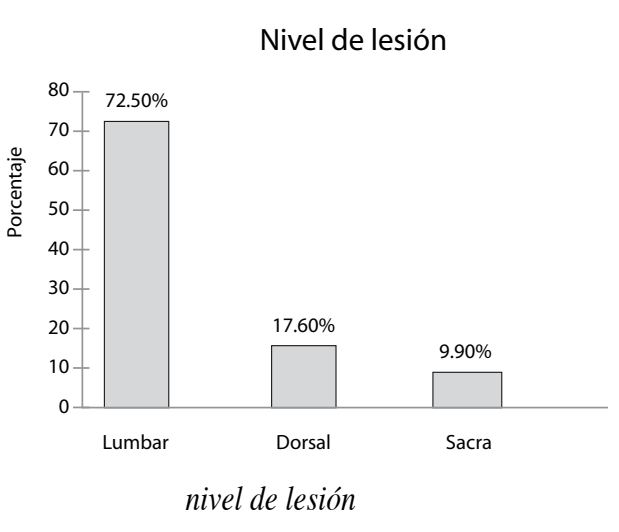

Gráfica 2. Tipo o nivel de lesión en 91 pacientes con mielomeningocele del CRIT Aguascalientes de enero a diciembre de 2008. 
Los pacientes con lesión lumbar presentaron desnutrición leve en el $12.1 \%$, sobrepeso $12.1 \%$, en $16.7 \%$ obesidad grado I, en $7.6 \%$ obesidad grado II, el $6.1 \%$ obesi- dad grado III, $12.1 \%$ obesidad mórbida y en el 33.3\% presentaron peso normal para la edad y talla. (Gráfica 3 y 4)

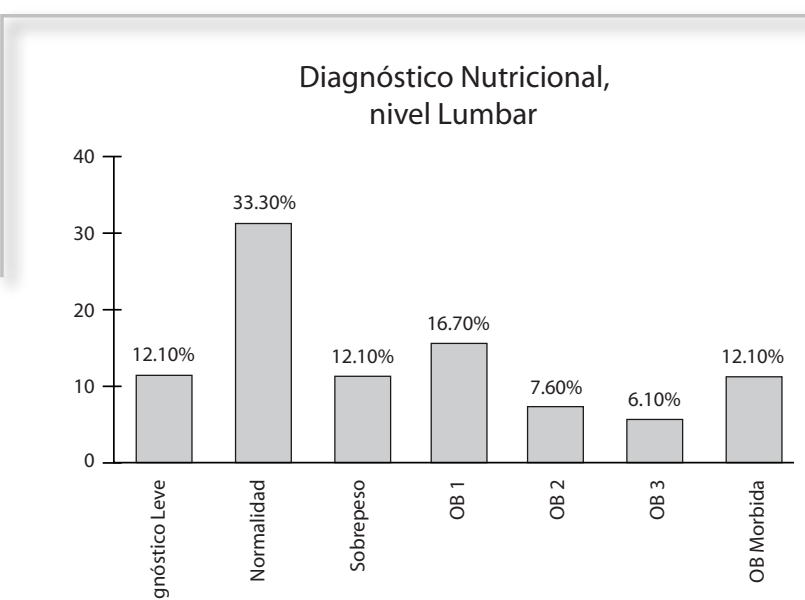

Gráfica 3. Diagnóstico nutricional en pacientes con afectación a nivel lumbar (DN=Desnutrición, $O B=$ Obesidad)
Diagnóstico Nutricional, nivel Lumbar

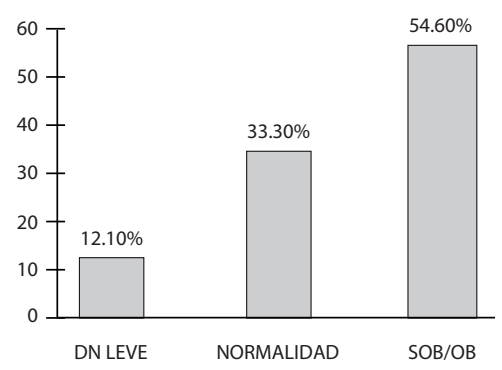

Gráfica 4. Diagnóstico nutricional en 91 pacientes con mielomeningocele del CRIT Aguascalientes con afección lumbar (DN=Desnutrición, $S O B=$ Sobrepeso, $O B=$ Obesidad)
Los pacientes con lesión dorsal; un paciente $(6.3 \%)$ presentó desnutrición leve, $5(31.3 \%)$ tuvieron sobrepeso, 4 (25\%) presentaron obesidad grado I, 3(18.8\%) con obesidad grado II y $3(18.8 \%)$ presentaron peso normal.(Gráfica 6)
En los pacientes con lesión sacra, únicamente un paciente $(11.1 \%)$ presentó sobrepeso y el resto de los pacientes 8 $(88.9 \%)$ presentaron peso normal. (Gráfica 7)
Diagnóstico Nutricional, nivel Dorsal

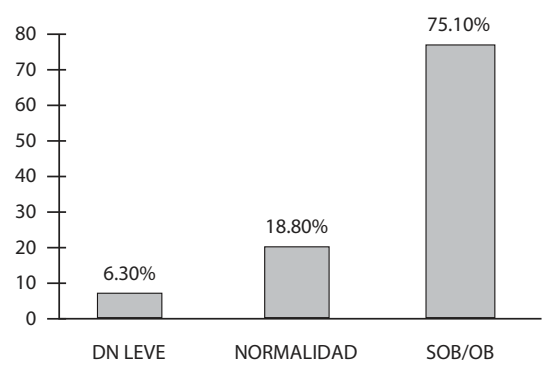

Gráfica 5. Diagnóstico nutricional en 91 pacientes con mielomeningocele del CRIT Aguascalientes con afección dorsal ( $D N=$ Desnutrición, $S O B=$ Sobrepeso, $O B=$ Obesidad)
Diagnóstico Nutricional, nivel Sacro

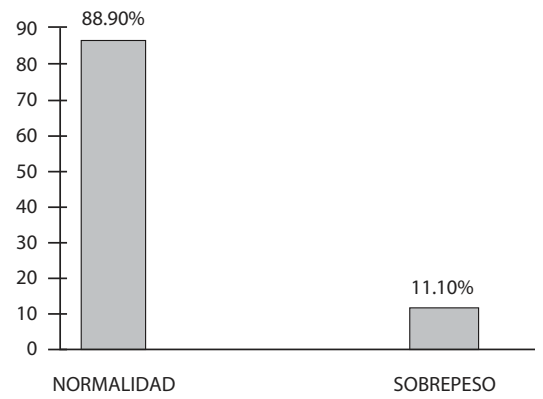

Gráfica 6. Diagnóstico nutricional en 91 pacientes con mielomeningocele del CRIT Aguascalientes con afección sacra. 


\section{Discusión}

Del total de pacientes evaluados, la mayoría presentaron lesión a nivel lumbar $(36.3 \%)$, seguida de lesiones dorsales (17.6\%) y por último, los pacientes con lesión sacra $(9.9 \%)$. Los resultados de este estudio en general coinciden con lo descrito por otros autores, sin embargo hay que resaltar que el nivel con alteraciones nutricias no fue precisamente en el grupo de lesión dorsal, como ha sido reportado en otros artículos ${ }^{3,4}$, sino en el grupo de afección a nivel lumbar en quienes encontramos grados extremos de obesidad, los cuales no se presentaron en la población de pacientes con afección a nivel de columna dorsal, si bien fue en estos últimos, en los que proporcionalmente se encontró mayor sobrepeso y obesidad.

El 38.5\% de la población estudiada fue diagnosticada con algún grado de obesidad, lo que concuerda con el estudio realizado por Torres y colaboradores, en el que se encontró que el $35 \%$ de su población también presentaba algún grado de obesidad. ${ }^{5}$

En los pacientes con lesión lumbar la prevalencia de sobrepeso y obesidad fue de $54.8 \%$ siendo en ellos en los únicos que se registraron diagnósticos de obesidad mórbida (12.1\%). Se debe considerar que estos pacientes representaron el $72.5 \%$ de los pacientes que se incluyeron en el estudio, que es similar a la población de pacientes que fue estudiada por Buffart y colaboradores en 2008 donde el $70 \%$ de su población presentó la lesión a nivel lumbar $^{6}$. Pudiera ser que al tener un porcentaje mayor de pacientes con lesión lumbar en nuestra muestra los grados de obesidad estén más elevados en ellos, pero proporcionalmente se registraron los índices más altos de obesidad en la población dorsal. ${ }^{5}$

En los pacientes con lesión dorsal los diagnósticos iban desde desnutrición leve hasta obesidad de tipo 2, en estos pacientes al presentar el nivel de la lesión más alto se esperaba encontrar alteraciones nutricias más graves, como grados de obesidad extremos, los cuales solo se presentaron en la población de lumbares, considerando que a mayor nivel de lesión aumenta el riesgo de obesidad, no obstante, también es posible que influyeran el mayor número de patologías concomitantes que presentan aunque nosotros no registramos este dato.

En cuanto a los pacientes con lesión sacra el $88.9 \%$ resultaron eutróficos y solo un $11.1 \%$ presentó sobrepeso, lo que se puede comparar con diversos estudios ${ }^{7-13}$ en los que se evaluaba el nivel de actividad física y se relacionaba con la génesis de la obesidad, en ellos cuya lesión es más baja y por tanto no se encuentra tan restringida su movilidad la tendencia a la obesidad disminuye $\mathrm{e}^{7-13}$.

Este estudio representa un diagnóstico inicial, que permitirá medir el impacto de la situación nutricia en los pacientes con respecto a sus hábitos alimenticios, actividad física, estado nutricional y calidad de vida.

La reducción de la actividad física en los niños con lesión medular también viene acompañada de un decremento en las necesidades energéticas de los pacientes, así, para que se pueda mantener un peso adecuado según la edad y talla, se deben adoptar hábitos de vida saludables que se adecúen a sus necesidades reales, en cuanto a requerimientos y actividad física, según la discapacidad con el fin de lograr un estado nutricional adecuado, conservar una óptima función cardiovascular y por consiguiente una mejor calidad y expectativa de vida ${ }^{14}$.

La orientación nutricional forma parte de un tratamiento integral que debe imperativamente promover en toda la familia buenos hábitos alimenticios, ya que es finalmente en el hogar donde el paciente logrará los objetivos nutricionales propuestos. 


\section{Conclusiones}

En la población estudiada, el 63.7\% presenta alteraciones en el estado de nutrición, siendo la más frecuente la obesidad y solamente la tercera parte de estos pacientes se encuentra con un peso dentro de la normalidad.

\section{Bibliografía}

1 Meix JM. Espina Bífida. Hospital Ramón y Cajal Madrid. Asociación Española de Pediatría. Disponible en:

http://www.sld.cu/galerias/pdf/sitios/rehabilitacion-temprana/espina_bifida.pdf

2 InfoFamilia. El portal Médico para padres de la asociación española de pediatría. Noviembre 2008. Obesidad Infantil.

3 Medina-Salas A, Coutiño-León B, Alvarado-Jiménez G, Ramírez -Ramírez J. Epidemiología del mielomeningocele en niños menores de un año de edad en el Instituto Nacional de Pediatría. Rev Mex Med Fís y Rehabil. 2001:13:50-54

4 Torres-González EM, MC Lara-Muñoz, CamachoGutiérrez S, González-Palafox MA. Factores psicosociales asociados al desarrollo de niños con mielomeningocele. Salud Mental. 2002; 25(6):44-52

5 Fiore $P$, Picco $P$, Castagnola E, Palmieri A, Levato $L$, Gremmo M. Nutritional Survey of children and adolescents with myelomenigocele (MMC): overweight associated with reduced energy intake. Eur J Pediatr Surg. 1998;8, S1:34-36

6 Buffart LM, Roebroeck ME, Rol M, Stam HJ, van den Berg-Emons RJ. Tried of physical activity, aerobic fitness and obesity in adolescents and young adults with myelomenigocele. J Rehabil Med. 2008;40(1):70-75.

7 Liusuwan RA, Widman LM, Abresch RT, Johnson AJ, McDonald CM. Behavioral Intervention, Exercise, and Nutrition Education to Improve Health and Fitness (BENEfit) in Adolescents With Mobility Impairment Due to Spinal Cord Dysfunction J Spinal Cord Med. 2007;30:S1:S119-S126.

8 Cañete-Estrada R, Cifuentes-Sabio V. Valoración del estado nutricional.

http://www.seep.es/privado/download.asp?url=/ publicaciones/2000TCA/Cap01.

9 Patiño M, Rojas Y. Manejo nutricional de patologías neurológicas. http://www.dynabizvenezuela.com/images/dynabiz/ID3749/siteinfo/NEURO2.

10 Sánchez-Castillo $C P$, Pichardo Ontiveros $E_{\text {, Ló- }}$ pez R. Epidemiología de la obesidad.http://www. anmm.org. $\mathrm{mx} /$ descargas/gaceta/suplementos/ Gmm_v140_s2/internet/PDF/2004-140-SUP2-320.pdf

11 Agencia española de seguridad alimentaria y nutrición. Evaluación del Índice de Masa Corporal en Niños y Niñas.

http://www.perseo.aesan.msps.es/docs/docs/ imc/evaluacion_imc_ninos.pdf

12 Shepherd K, Roberts D, Golding S, Thomas BJ, Sheperd RW. Body composition in myelomeningocele. American J Clin Nut. 1991;53(1):1-6

13 Jacobs RA, Blyler E, Baer MT. Nutrition risk factors in children with myelomeningocele. Eur J Pediatr Surg. 1991;S1,22

14 Buffart LM, van den Berg-Emons RJ, van WijlenHempel MS, Stam HJ, Roebroeck ME. Health-related physical fitness of adolescents and young adults with myelomeningocele. Eur J Appl Physiol. 2008;103(2):181-8

Mi agradecimiento al Centro de Rehabilitación Infantil Teletón, que me permitió realizar mi servicio en esta institución, dándome así las herramientas para elaborar este estudio. Le agradezco también a mi asesor y amigo el Maestro Adán Rodríguez por todo el apoyo brindado, por inspirarme a ser mejor cada día y por su constancia y dedicación. 